\title{
Produção de agregado sintético de argila com reaproveitamento de resíduo de vidro
}

\section{Production of synthetic clay aggregate with reuse of glass residue}

\author{
Herbet Alves de Oliveira ${ }^{1}$, Cochiran Pereira dos Santos ${ }^{2}$; \\ Rosane Maria Pessoa Betânio Oliveira ${ }^{1,4}$, Edilson de Jesus ${ }^{3}$, \\ Zélia Soares Macedo ${ }^{1,2}$
}

\footnotetext{
${ }^{1}$ Programa de Pós-graduação em Ciência e Engenharia de Materiais - P²CEM/UFS, CEP: 49100-000, São Cristóvão, SE, Brasil.

${ }^{2}$ Departamento de Física - DFI/UFS, CEP: 49100-000, São Cristóvão, Sergipe, Brasil.

${ }^{3}$ Departamento de Engenharia Química - DQI/UFS, CEP: 49100-000, São Cristóvão, Sergipe, Brasil.

${ }^{4}$ Departamento de Ciência e Engenharia de Materiais - DCEM/UFS, CEP: 49100-000, São Cristóvão, Sergipe, Brasil. e-mail: herbetalves148@gmail.com, cochiran@hotmail.com, rosaneboliveira@gmail.com, Zélia.macedo@gmail.com
}

\section{RESUMO}

É objetivo da construção civil, reduzir o peso das estruturas e consequentemente os custos com as fundações. A substituição de britas por agregados leves é a alternativa mais utilizada. No entanto, sobretudo no Brasil, só existe uma única fábrica de agregados leves localizada em São Paulo, sendo oportuna a pesquisa de matériasprimas para disseminação da produção de agregado em outras regiões do Brasil. Proporcionalmente, esse tipo de segmento valoriza o reaproveitamento de resíduos de diversos segmentos. Neste trabalho foi produzido um agregado sintético de argila calcinada com a incorporação de resíduo de vidro de embalagem à base de $\mathrm{SiO}_{2}, \mathrm{Na}_{2} \mathrm{O}$ e $\mathrm{CaO}$, com o intuito de substituir britas para uso em concreto. Foram testadas incorporações de 0 a $20 \%$ de vidro e as massas foram caracterizadas por índice de plasticidade, DRX, FRX, DTA/TG, capacidade de troca de cátions e massa específica real. Os corpos de prova foram produzidos por prensagem axial a uma pressão de $30 \mathrm{MPa}$, em formato cilíndrico com $20 \mathrm{~mm}$ de diâmetro e altura de $15 \mathrm{~mm}$. Após queima, os corpos de prova foram caracterizados em ensaios de absorção de água, densidade aparente e resistência mecânica à compressão. Foi observado que à medida que o resíduo de vidro foi incorporado nas formulações, as misturas apresentaram resistência mecânica cada vez maior e absorção de água cada vez menor, devido a incorporação dos óxidos fundentes que favoreceram a formação de fase liquida. No entanto, quanto se atingiu o limite de incorporação de $20 \%$ e queima a $1000{ }^{\circ} \mathrm{C}$, foi observado um aumento de volume devido a maior formação de fase líquida que preencheu os espaços vazios dificultando a saída dos gases que ficaram aprisionados. Concluiu-se que o resíduo de vidro quando incorporado na argila em estudo pode ser utilizada de duas formas: como fundente para acréscimo da resistência mecânica e massa especifica aparente e redução da absorção de água ou ainda com a função de redução da densidade propriedade que é fundamental para produção de agregados leves.

Palavras-chave: agregado sintético, cerâmica, vidro, reciclagem.

\section{ABSTRACT}

it is the objective of the civil construction, to reduce the weight of the structures and consequently the costs with the foundations. The replacement of brittle by light aggregates is the most commonly used alternative. However, especially in Brazil, there is only a single light aggregate plant located in São Paulo, and the timing of research on raw materials for the dissemination of aggregate production in other regions of Brazil. Proportionally, this type of segment values the reuse of waste from several segments. In this work a synthetic aggregate of calcined clay was produced with the incorporation of glass residue of packing with $\mathrm{SiO}_{2}, \mathrm{Na}_{2} \mathrm{O}$ and $\mathrm{CaO}$ base, in order to replace brittle for concrete use. Incorporations of 0 to $20 \%$ of glass were tested and 
the masses were characterized by plasticity index, XRD, FRX, DTA / TG, cation exchange capacity and actual specific mass. The specimens were produced by axial pressing at a pressure of $30 \mathrm{MPa}$, in a cylindrical format with a diameter of $20 \mathrm{~mm}$ and a height of $15 \mathrm{~mm}$. After burning, the specimens were characterized in tests of water absorption, apparent density and mechanical resistance to compression. It was observed that as the glass residue was incorporated into the formulations, the mixtures presented increasing mechanical strength and lower water absorption due to the incorporation of the fluxing oxides that favored the formation of liquid phase. However, when the $20 \%$ incorporation limit was reached and the burn at $1000 \mathrm{oC}$, a volume increase was observed due to the higher liquid phase formation that filled the void spaces, making it difficult to escape the gases trapped. It was concluded that the glass residue when incorporated in the clay under study can be used in two ways: as a flux for addition of mechanical resistance and apparent specific mass and reduction of water absorption or with the function of reducing the property density that is fundamental for the production of lightweight aggregate.

Keywords: synthetic aggregate, ceramics, glass, recycling.

\section{INTRODUÇÃO}

Os agregados leves podem apresentar massa unitária que pode chegar a $2000 \mathrm{~kg} / \mathrm{m}^{3}$ enquanto nos agregados pesados a densidade ultrapassa $2000 \mathrm{~kg} / \mathrm{cm}^{3}$ [1]. Os agregados leves têm ampla aplicação na fabricação de concreto com o fim de redução do peso da estrutura ou como elemento de proteção acústica [2,3], ou ainda tem aplicação em pavimentação asfáltica [4]. Em estudos recentes, o agregado sintético calcinado tem sido investigado para utilização em concreto para atender a necessidade de regiões em que a brita não está disponível [5,6]. De modo geral, na produção de agregados é valorizado o reaproveitamento de resíduos diversos. O processamento de resíduos industriais é um tema importante não só do ponto de vista para redução da poluição ambiental perigosa, mas também devido à potencialidade para reaproveitamento [7, 8]. Diversos autores têm se empenhado na pesquisa de reaproveitamento de resíduos, como vidros, cinzas e lamas diversas [7, $9,10,11,12]$.

Segundo a Associação Brasileira de Empresas de Limpeza Pública e Resíduos Especiais [13], só no Brasil são geradas 1.332.827 ton/ano de resíduos de vidro de embalagem correspondente a 2\% dos resíduos urbanos, sendo que $20 \%$ do total são descartados em lixo. O vidro é uma substância inorgânica, homogênea e amorfa, obtida através do resfriamento de uma massa proveniente de uma mistura de matérias-primas à base de sílica fundida e resfriada. $\mathrm{O}$ vidro distingue-se de outros materiais por várias características: não é poroso nem absorvente, é ótimo isolador e possui baixo índice de dilatação. O vidro de embalagem quando descartado, pode ser uma alternativa para reaproveitamento. Na produção de cerâmicas, sobretudo agregados de argila, é valorizado o reaproveitamento de resíduos de vidro como fonte de fundentes. Por sua vez, o vidro reduz drasticamente a viscosidade quando misturado em massas cerâmicas, dificultando obter produtos com baixa absorção de água e elevada resistência mecânica devido à deformação que normalmente ocorre. O vidro de embalagem possui composição química à base de $\mathrm{Na}_{2} \mathrm{O}$ e $\mathrm{CaO}$, que são óxidos fundentes e por isso reaproveitável na produção de cimento, concretos e argamassas, além de poder ser reciclado no próprio processo produtivo do vidro $[14,15]$.

Pesquisadores têm investigado o uso do vidro em formulações cerâmicas com a finalidade de reduzir a temperatura de queima e melhorar suas propriedades $[16,17,18,19]$. O principal desafio na utilização do vidro é ajustar os parâmetros de produção de modo a evitar a deformação sem a completa densificação da peça, o que é bastante comum devido à baixa viscosidade obtida, sobretudo devido ao enriquecimento da formulação pelos íons alcalinos [20].

Neste trabalho, foram selecionadas três argilas utilizadas nos segmentos de revestimento cerâmico e cerâmica vermelha do estado de Sergipe e Alagoas, Brasil, as quais foram utilizadas para elaborar formulações e definido por meio dos ensaios tecnológicos a absorção de água, resistência mecânica à compressão e massa específica aparente, a formulação que apresentou os resultados mais próximos de uma brita [21]. Assim sendo, o objetivo específico foi produzir agregado leve a partir de argilas do segmento de cerâmico em Sergipe e introduzir resíduo de vidro contribuindo para a redução do descarte do mesmo.

\section{MATERIAIS E MÉTODOS}

\subsection{Material}

As argilas investigadas apresentadas nesse trabalho são provenientes de duas jazidas localizadas no estado de Sergipe e uma em Alagoas, região nordeste do Brasil. A argila Pinheiro (1-PIN) é proveniente do município de Laranjeiras (SE) de coordenadas geográficas Latitude: $10^{\circ} 48^{\prime} 12^{\prime \prime}$ Sul e Longitude: $37^{\circ} 10^{\prime} 11^{\prime \prime}$ Oeste; a 
argila Igreja Nova (2-IN) é proveniente do município de Igreja Nova (AL) de Latitude: 10 7' 13" Sul, Longitude: $36^{\circ} 39^{\prime} 39^{\prime \prime}$ Oeste. A argila (3-MA) Morro Alto, é proveniente do município de Itabaianinha (SE) de Latitude: $11^{\circ} 16^{\prime} 27^{\prime \prime}$ Sul, Longitude: $37^{\circ} 47^{\prime} 20^{\prime \prime}$ Oeste. O resíduo de vidro utilizado foram embalagens descartadas de cor incolor.

\subsection{Preparação das formulações e testes de caracterização}

Uma amostra de aproximadamente $50 \mathrm{~kg}$ de cada argila foi colhida diretamente dos depósitos das jazidas para realização dos ensaios. As amostras foram homogeneizadas, secas ao ar e depois em estufa a temperaturas de $(40 \pm 10){ }^{\circ} \mathrm{C}$ por $48 \mathrm{~h}$. Para iniciar a caracterização tecnológica destes materiais, foi realizada sua desagregação e redução granulométrica por meio de moinho de martelo com abertura de grelha de $2 \mathrm{~mm}$. Uma amostra de cerca de $1 \mathrm{~kg}$ foi separada de cada formulação por quarteamento e passada em peneira \#200 $(0,074 \mathrm{~mm})$ para realização dos ensaios de caracterização: difratometria de raios X-DRX, análise química por fluorescência de raios X-FRX, análise granulométrica, índice de plasticidade, capacidade de troca de cátions e análise térmica DTA/TG. Foi ainda coletada outra amostra de cerca de $2 \mathrm{~kg}$ e passada em peneira \# 60 $(0,25 \mathrm{~mm})$ para produção de corpos de prova outra amostra de cerca de $2 \mathrm{~kg}$ e passada em peneira \# $60(0,25$ $\mathrm{mm})$ para produção de corpos de prova. As amostras na forma de pó, passadas na peneira \# $60(0,25 \mathrm{~mm})$, foram umidificadas e granuladas utilizando um teor de água de $8 \%$ e passadas na peneira \# $16(1,2 \mathrm{~mm})$ para ajuste da granulometria. Em seguida, as formulações foram deixadas em descanso por $24 \mathrm{~h}$ para homogeneização da umidade. O resíduo de vidro foi moído em moinho de bolas e passado na peneira \#325 $(0,044 \mathrm{~mm})$ [22].

Foram conformados corpos de prova cilíndricos com dimensões 20 x $20 \mathrm{~mm}$ em uma prensa manual da marca Nowak com pressão uniaxial de compactação de $35 \mathrm{MPa}$. Os corpos de prova foram secos inicialmente ao ar livre por $24 \mathrm{~h}$ e depois em estufa a $(100 \pm 5){ }^{\circ} \mathrm{C}$ até peso constante.

A etapa de queima foi realizada em forno da marca JUNG modelo LF 0612 nas temperaturas de 870, $950,1000,1080$ e $1100{ }^{\circ} \mathrm{C}$ para a uma taxa de $20{ }^{\circ} \mathrm{C} / \mathrm{min}$ com patamar de 20 min na maior temperatura. Após queima, os corpos de prova foram caracterizados por meio da absorção de água $(A A)$ com imersão em água fervente por $2 \mathrm{~h}$ segundo a norma ASTM C-20-2005 [23] cujos resultados podem ser expressos pela Equação (1), em que $m_{1}$ é a massa seca e $m_{2}$ a massa saturada:

$$
A A=\left(m_{2}-m_{1} / m_{1}\right) \cdot 100
$$

Foi ainda determinada a massa específica aparente (Meaq) dos corpos sinterizados, usando o método de Arquimedes [22].

A tensão de ruptura à compressão (TRC), após queima, foi obtida em tensiômetro da marca INSTRON, modelo $3385 \mathrm{H}$, com velocidade de aplicação de carga de $1 \mathrm{~mm} / \mathrm{min}$, utilizando corpos de prova cilíndricos, cujos resultados podem ser expressos pela Eq. (2) em que P é a carga aplicada em N, e A é a área da seção transversal $\left(\mathrm{mm}^{2}\right)$ adaptado da Norma NBR 10832 [24]

$$
\mathrm{TRC}=\mathrm{P} / \mathrm{A}
$$

\subsection{Análise química}

Os percentuais dos óxidos maiores constituintes das amostras foram determinados através de medidas semiquantitativas pela técnica de fluorescência de raios X (FRX). As medidas foram realizadas em vácuo, em um equipamento da marca Bruker, modelo S4 Pioneer, utilizando amostras com massa em torno de $10 \mathrm{~g}$ que foram prensadas no formato de corpos cilíndricos com diâmetro de $20 \mathrm{~mm}$ e espessura de $3 \mathrm{~mm}$, aproximadamente.

\subsection{Perda ao fogo}

Foi realizada pela diferença das massas antes e após a queima a $1000{ }^{\circ} \mathrm{C}$ em forno com patamar de $2 \mathrm{~h}$ [25]. 


\subsection{Capacidade de troca de cátions}

A troca catiônica foi determinada pelo método do azul de metileno de acordo com a norma ASTM 837-1992 [26].

\subsection{Análise térmica diferencial e gravimétrica (DTA-TG)}

Os eventos térmicos apresentados pela amostra no intervalo de temperatura entre 25 e $1200{ }^{\circ} \mathrm{C}$ foram registrados em medidas simultâneas de DTA e TG. As medidas foram realizadas em um equipamento da TA Instruments, modelo SDT 2960. As amostras foram medidas em cadinho de platina, sob fluxo de ar sintético com vazão de $100 \mathrm{~mL} / \mathrm{min}$ e taxa de aquecimento de $10^{\circ} \mathrm{C} / \mathrm{min}$.

\subsection{Avaliação da plasticidade}

Os limites de liquidez (LL) e de plasticidade (LP) foram obtidos de acordo com a norma ASTM D4318 [27] e NBR 6459 [28]. O índice de plasticidade (IP) é o resultado da diferença aritmética entre os limites de liquidez e plasticidade, o qual pode ser expresso pela Equação (3), em que IP é o índice de plasticidade, LL é o limite de liquidez e LP é o limite de plasticidade. O erro experimental foi de aproximadamente $\pm 3 \%$.

$$
I P=L L-L P
$$

\subsection{Análise mineralógica}

A difratometria de raios $\mathrm{X}$ foi utilizada para a identificação das fases cristalinas de acordo com os padrões obtidos no banco de dados do ICSD (Inorganic Crystal Structure Database) e a análise foi realizada utilizando o software Match. Os padrões de difração foram obtidos em um equipamento Rigaku D-MAX 100 usando radiação $\mathrm{Cu} \mathrm{K}_{\alpha 1}\left(\lambda=1,5418 \AA\right.$ ) em modo de varredura contínua, em intervalo angular de 5 a $70^{\circ}$ com velocidade de varredura de $1 \%$ min. Para confirmação da fase montmorilonita uma amostra foi saturada com etileno glicol por $1 \mathrm{~h}$ afim de observar o aumento da distância interplanar e outra amostra foi calcinada a $550{ }^{\circ} \mathrm{C}$ por 2 h. Em seguida, ambas foram analisadas por DRX, no intervalo de varredura de 2 a $15^{\circ}$ [25]. A análise foi realizada em duas partes: a fração argila $(<2 \mu \mathrm{m})$ foi separada por centrifugação, em seguida tratada com $\mathrm{H}_{2} \mathrm{O}_{2}$ para eliminação de matéria orgânica e com $\mathrm{HCl}$ para eliminação de carbonatos. Com o material restante, analisou-se os acessórios [29].

\subsection{Análise dilatométrica}

As medidas dilatométricas foram realizadas para verificar as alterações dimensionais de expansão e retração térmica envolvidas no processo de densificação das amostras. Os corpos de prova foram preparados por compactação em um molde cilíndrico de 12 x $6 \mathrm{~mm}$ de dimensão e previamente calcinados a $440{ }^{\circ} \mathrm{C}$ por $2 \mathrm{~h}$ para eliminação da matéria orgânica. Nos ensaios, foi utilizado um dilatômetro da marca Netzsch DIL 402PC com fluxo de ar sintético e vazão de $100 \mathrm{~mL} / \mathrm{min}$, variando da temperatura ambiente até $1200{ }^{\circ} \mathrm{C}$ com taxa de aquecimento de $10{ }^{\circ} \mathrm{C} / \mathrm{min}$. A temperatura ideal de queima foi obtida a partir da derivada da curva dilatométrica, correspondendo à temperatura de máxima retração.

\subsection{Medida da densidade}

O processo de densificação foi determinado a partir dos valores de retração linear obtida nos ensaios de dilatometria, de acordo com a Equação (4), em que $\rho_{o}$ é a densidade dos corpos de prova pré calcinados, determinada tomando-se as medidas de comprimento, diâmetro e massa. $\mathrm{L}_{\mathrm{o}}$ é o comprimento inicial dos corpos de prova e $\Delta L$ é a variação dimensional em função da temperatura [30]:

$$
\rho=\rho_{0}\left(1-\Delta L / L_{0}\right)^{3}
$$

\subsection{Massa específica}

As medidas de massa específica das formulações em pó foram feitas por picnometria a hélio em um picnômetro da marca Micromeritics, modelo Accupyc 1330. 


\section{RESULTADOS}

A análise de difratometria de raios X das argilas utilizadas no trabalho é apresentada na Figura 1. As argilas apresentam majoritariamente os argilominerais illita (JCPDS 96900-9666) que fornece o óxido $\mathrm{K}_{2} \mathrm{O}$ que favorece a sinterização durante a queima. Em menor proporção, foram observadas as fases montmorillonita (JCPDS 96901-0959) na argila 1-PIN, que apresenta elevada plasticidade [29]. O difratograma apresenta ainda o acessório muscovita (JCPDS 96101-1059) em todas as argilas, que fornece os óxidos $\mathrm{Na}_{2} \mathrm{O}$ e $\mathrm{K}_{2} \mathrm{O}$, além do quartzo (JCPDS 96101-1160), que juntamente com os demais óxidos forma fase vítrea que preenche os poros, conferindo a densificação da massa após queima [31].

Foi observada ainda a presença da calcita (JCPDS 96702-0140) na argila 1-PIN que após dissociação fornece o $\mathrm{CaO}$. Foi realizada também análise difratométrica da amostra 1-PIN após tratamento com etileno glicol e calcinada a $550{ }^{\circ} \mathrm{C}$ por $2 \mathrm{~h}$ [32]. Após adição do etileno glicol, o espaçamento basal da montmorillonita foi deslocado da distância interplanar 14,3 $\AA$ para 16,4 $\AA$, determinado através da lei de Bragg: $n \lambda=$ $2 d \operatorname{sen} \theta$, confirmando a presença da montmorillonita na argila 1-PIN [25].

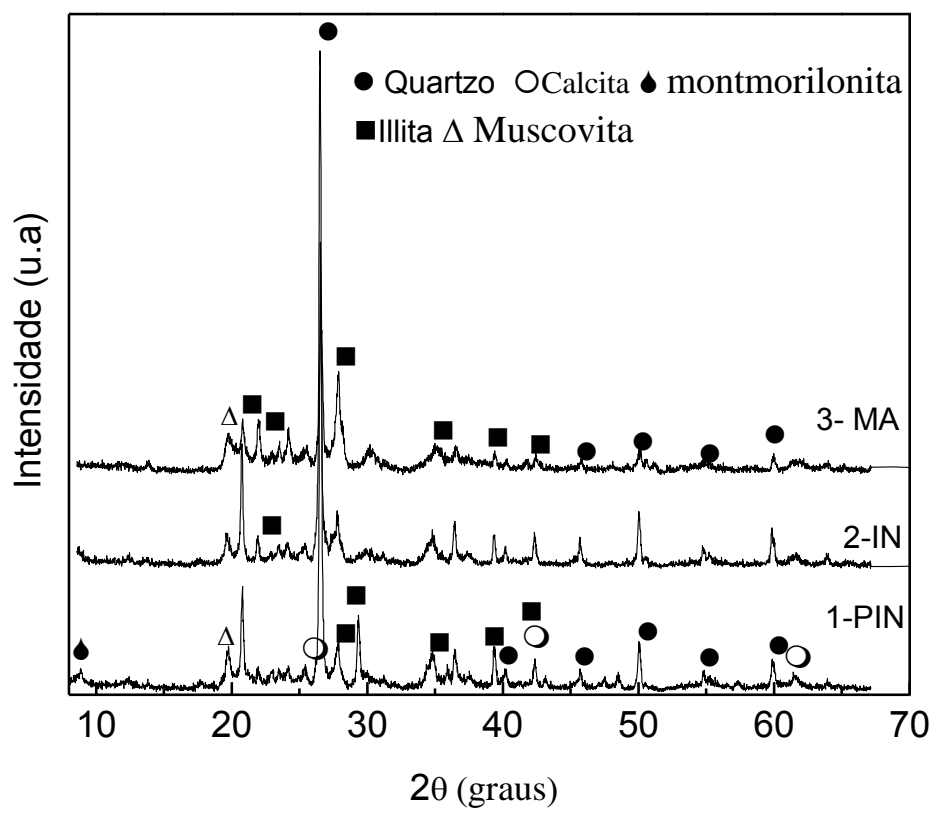

Figura 1: Padrões de difração de raios $\mathrm{X}$ das argilas estudadas.

A Tabela 1 mostra os resultados da análise química das argilas investigadas e do resíduo de vidro. As argilas são constituídas majoritariamente pela $\mathrm{SiO}_{2}$ e $\mathrm{Al}_{2} \mathrm{O}_{3}$, seguido dos álcalis $\mathrm{K}_{2} \mathrm{O}$ e $\mathrm{Na}_{2} \mathrm{O}$ além de consideravel teor de $\mathrm{Fe}_{2} \mathrm{O}_{3}$, os quais são elementos associados com estruturas de argilominerais, quartzo e feldspatos, típicos de produtos de cerâmica vermelha [34]. O teor de $\mathrm{SiO}_{2}$ oscilou de 53,7 a 65,2\%, enquanto os teores de $\mathrm{Al}_{2} \mathrm{O}_{3}$ de 16,5 a 17,1\%. A relação $\left(\mathrm{SiO}_{2} / \mathrm{Al}_{2} \mathrm{O}_{3}\right)$ variou entre valores de 3,25 a 4,26 os quais são mais altos do que os valores clássicos para a caulinita $\left(\mathrm{Si}_{2} / \mathrm{Al}_{2} \mathrm{O}_{3}: 1,18\right)$ ou para a montmorillonita $\left(\mathrm{SiO}_{2} / \mathrm{Al}_{2} \mathrm{O}_{3}\right.$ : 2,36) [34], indicando que quanto maior este coeficiente, maior é a porcentagem de quartzo livre e mineral acessório presentes nas argilas. Os teores de óxidos alcalinos $\left(\mathrm{Na}_{2} \mathrm{O}+\mathrm{K}_{2} \mathrm{O}\right)$ têm ação fundente e estão de acordo com outros trabalhos [32,33].

A maior porcentagem de óxidos fundentes foi observada na argila 2-IN (5,9\%) que favorece a formação de fase líquida no processo de queima, contribuindo para a redução de poros e aumentando a densificação dos materiais. Por sua vez, o elevado teor de $\mathrm{CaO}$ observado na argila 1-PIN (7\%), é uma indicação que existem carbonatos que são prejudiciais ao fenômeno de densificação [35]. Adicionalmente, o resíduo de vidro é rico em álcalis, os quais favorecem a sinterização da massa [34,35].

Tabela 1: Composição química das argilas determinada por fluorescência de raios X (FRX) (\%) 


\begin{tabular}{c|c|c|c|c|c|c|c|c|c|c|c|c}
\hline Óxido & $\mathrm{SiO}_{2}$ & $\mathrm{Al}_{2} \mathrm{O}_{3}$ & $\mathrm{Fe}_{2} \mathrm{O}_{3}$ & $\mathrm{CaO}$ & $\mathrm{K}_{2} \mathrm{O}$ & $\mathrm{Na}_{2} \mathrm{O}$ & $\mathrm{MgO}$ & $\mathrm{Cl}$ & $\mathrm{TiO}_{2}$ & $\mathrm{PF}$ & $\begin{array}{c}\mathrm{Total} \\
\mathrm{Na}_{2} \mathrm{O} \\
+\mathrm{K}_{2} \mathrm{O}\end{array}$ \\
\hline 1-PIN & 53,7 & 16,0 & 6,6 & 7,0 & 3,2 & 1,3 & 2,7 & 0,2 & 0,0 & 8,8 & 100 & 4,5 \\
\hline 2-IN & 65,2 & 15,3 & 5,3 & 0,7 & 4,3 & 1,6 & 2,7 & 0,2 & 0,9 & 3,8 & 100 & 5,9 \\
\hline 3-MA & 62,3 & 17,1 & 5,6 & 2,4 & 1,6 & 2,9 & 1,6 & 0,3 & 0,7 & 5,0 & 100 & 4,5 \\
\hline $\begin{array}{c}\text { Resíduo } \\
\text { de vidro }\end{array}$ & 68,8 & 1,9 & 0,2 & 15,5 & 0,4 & 12,2 & 0,2 & 0,0 & 0,1 & 2,0 & 100 & 12,5 \\
\hline
\end{tabular}

A Figura 2 apresenta as curvas de DTA das argilas. Foi observado pico endotérmico a cerca de $100{ }^{\circ} \mathrm{C}$ em todas as argilas que segundo Celik [31], é perda de água adsorvida. Pode ocorrer ainda perda de hidroxilas nas argilas até $700{ }^{\circ} \mathrm{C}$ o que é comum. Foi observado na argila 1-PIN pico endotérmico a $720{ }^{\circ} \mathrm{C}$ devido a dissociação de carbonatos os quais dificultam a sinterização. Foi ainda identificado na argila 1-PIN, a 1150 ${ }^{\circ} \mathrm{C}$ e a $1100{ }^{\circ} \mathrm{C}$ na argila 2-IN e 3-MA, pico endotérmico devido a dissociação da sílica. A argila 3-MA foi a que apresentou maior risco para ruptura durante a queima em função do pico exotérmico a $580{ }^{\circ} \mathrm{C}$ devido a dissociação de matéria orgânica.

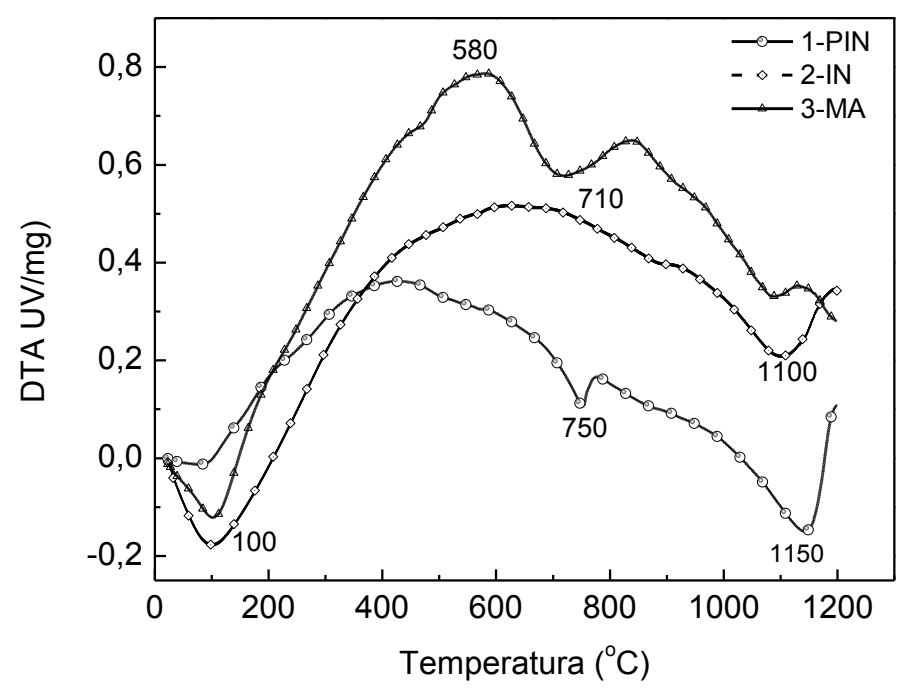

Figura 2: Análise Térmica Diferencial das formulações padrão com 10 e $20 \%$ de vidro.

Os valores de IP na Tabela 2 variaram de 9 a 16\%. A argila 2-IN apresenta baixo índice de plasticidade devido provavelmente ao elevado teor de quartzo e minerais acessórios como muscovita, identificados no difratograma de raios X na Figura 2. As argilas 1-PIN e 3-MA apresentaram plasticidade adequada para conformação cerâmica vermelha provavelmente devido à sua maior porcentagem de matéria orgânica conforme mostrado na Tabela 2. A distribuição granulométrica das argilas conforme discutido por [31] a fração de finos menores que $2 \mu \mathrm{m}$ confere maior área específica, favorecendo o desenvolvimento da plasticidade, obtendo-se maior resistência mecânica dos corpos conformados a verde e, proporcionalmente, melhora a sinterização e a resistência mecânica após queima. A argila 1-PIN apresentou maior área específica (ME) provavelmente devido a presença de maior porcentagem de acessórios como calcita. 
Tabela 2: Caracterização física das argilas

\begin{tabular}{l|c|c|c|c|c|c|c}
\hline Amostra & IP $(\%)$ & $\begin{array}{c}\text { Argila } \\
<2 \\
(\mu \mathrm{m})\end{array}$ & $\begin{array}{c}\text { Silte } \\
2-60 \\
(\mu \mathrm{m})\end{array}$ & $\begin{array}{c}\text { Areia } \\
>60 \\
(\mu \mathrm{m})\end{array}$ & $\begin{array}{c}\mathrm{CTC} \\
(\mathrm{meq} / 100)\end{array}$ & $\begin{array}{c}\text { MO } \\
(\%)\end{array}$ & $\begin{array}{c}\mathrm{ME} \\
\left(\mathrm{g} / \mathrm{cm}^{3}\right)\end{array}$ \\
\hline 1-PIN & 15 & 25,1 & 66,4 & 8,5 & $9,5 \pm 0,1$ & $1,8 \pm 0,1$ & $2,744 \pm 0,006$ \\
\hline 2-IN & 9 & 39,7 & 39,2 & 21,1 & $12,6 \pm 0,1$ & $1,4 \pm 0,2$ & $2,710 \pm 0,003$ \\
\hline 3-MA & 16 & 30,1 & 39,3 & 30,6 & $17,3 \pm 0,1$ & $3,4 \pm 0,1$ & $2,520 \pm 0,001$ \\
\hline
\end{tabular}

IP - índice de plasticidade; CTC - capacidade de troca de cátions; MO - matéria orgânica; ME - massa específica.

A Tabela 3 apresenta as proporções de matérias-primas em cada formulação investigada no presente trabalho. A mistura padrão não contém resíduos de vidro, enquanto nas demais foi incorporado o resíduo com o fim de introduzir óxidos fundentes para melhorar as propriedades mecânicas, reduzir a temperatura de queima e promover a reutilização do vidro como alternativa para redução do impacto ambiental.

Tabela 3: Composição estudada (\%).

\begin{tabular}{c|c|c|c|c}
\hline Matéria-prima & Padrão & $5 \%$ & $10 \%$ & $20 \%$ \\
\hline 2-IN & 60 & 57 & 54 & 48 \\
\hline 1-PIN & 20 & 19 & 18 & 16 \\
\hline 3-MA & 20 & 19 & 18 & 16 \\
\hline Resíduo de vidro & - & 5 & 10 & 20 \\
\hline
\end{tabular}

Na Figura 3 é apresentada a curva dilatométrica das massas estudadas, em que é apresentada a evolução da dilatação e da densidade em função da variação da temperatura. Foi observado que em todas as formulações à medida que a temperatura é aumentada, a densidade diminuiu até próximo de $700{ }^{\circ} \mathrm{C}$. A redução da densidade se deve ao aumento de volume provocado pela expansão decorrente da mudança da forma alotrópica do quartzo $\alpha$ para $\beta$ a $573{ }^{\circ} \mathrm{C}$ [33] e a cerca de $600{ }^{\circ} \mathrm{C}$, devido a desidroxilação das argilas, que contribuiu para a redução ainda mais da densidade [35].

Para temperaturas superiores a $700{ }^{\circ} \mathrm{C}$, a densidade aumenta e a sinterização ocorre com formação de fase líquida que preenche os poros abertos [25]. Na Figura 4 foi observado ainda, que a taxa máxima de retração foi atingida na massa padrão em $\mathrm{T}=1100{ }^{\circ} \mathrm{C}$. Para as amostras contendo 5,10 e $20 \%$, as temperaturas correspondendo à taxa máxima de retração foram 1050, 1020 e $950{ }^{\circ} \mathrm{C}$, respectivamente. Assim, à medida que o resíduo é acrescentado, diminui a temperatura ideal para sinterização, o que ocorre devido aos álcalis introduzidos. Após atingir a densidade máxima, ocorreu provavelmente o fenômeno da expansão (ou inchaço) o qual uma parte do material fundido retém os gases devido à decomposição dos minerais e a densidade tende a diminuir [11]. Estes gases são decorrentes da dissociação da matéria orgânica ou dissociação das argilas ou ainda carbonatos. $\mathrm{O} \mathrm{Fe}^{+2}$ presente nas argilas na presença de oxigênio tende a oxidar em $\mathrm{Fe}^{+3}$. A falta de oxigênio induz o $\mathrm{Fe}^{+3}$ a reduzir em $\mathrm{Fe}$ liberando oxigênio e provocando a expansão [35,37]. 


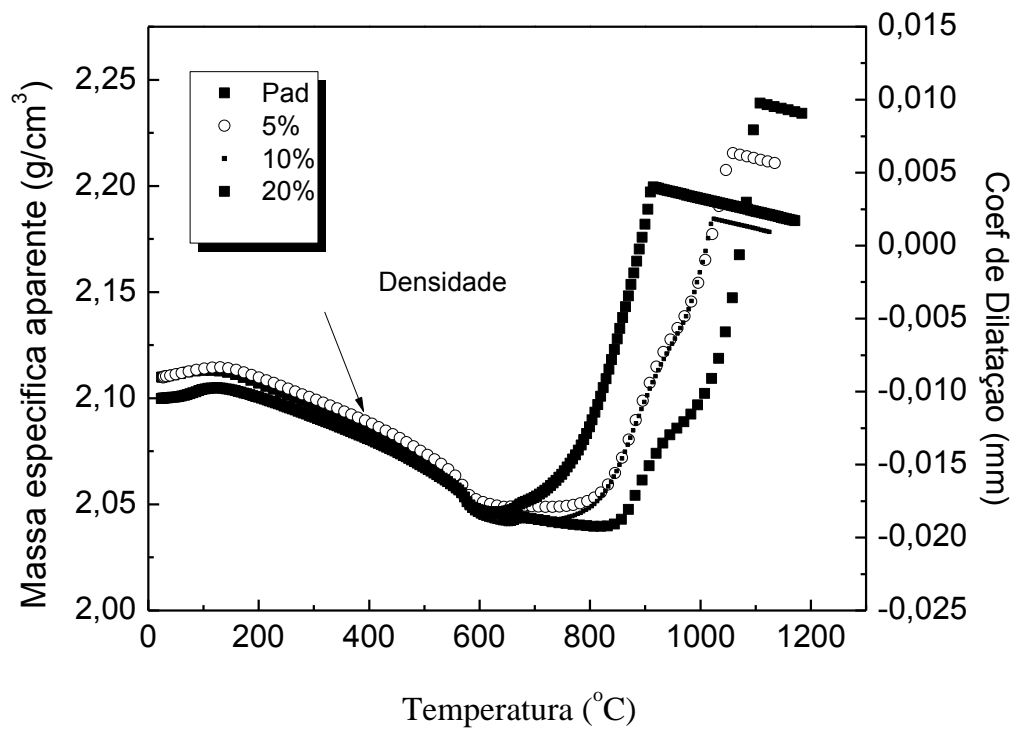

Figura 3: Densidade das amostras em função da temperatura de queima.

Nas Figuras 4 e 5 são apresentados os resultados dos ensaios tecnológicos de absorção de água, resistência mecânica à compressão e massa específica após queima nas temperaturas de 870, 950, 1000, 1080 e $1100{ }^{\circ} \mathrm{C}$, definidas pelos ensaios de análise dilatométrica. Em todos os casos, o patamar de queima foi de 20 min. Na Figura 4 é apresentada a absorção de água (AA) dos corpos de prova sinterizados. Para amostras contendo até $10 \%$ de vidro, observou-se que, à medida que se aumentou a temperatura até $1100{ }^{\circ} \mathrm{C}$, a absorção de água diminuiu. Os resultados obtidos a partir da incorporação de resíduo de vidro: padrão $(2,8 \%), 5 \%$ $(2,4 \%)$ e $10 \%(1,9 \%)$. A formulação contendo $20 \%$ de vidro apresentou redução bastante acentuada nos valores de absorção de água até o limite de $1000^{\circ} \mathrm{C}$, atingindo $2,8 \%$. No entanto, para temperaturas superiores a $1000{ }^{\circ} \mathrm{C}$ a absorção de água aumentou até $4,8 \%$, o que possivelmente ocorreu devido à expansão térmica da amostra nesta faixa de temperaturas devido a maior formação de fase liquida as quais preencheram os espaços vazios e provocou aprisionamento dos gases provocando expansão [11, 35]. De um modo geral, os resultados de absorção de água obtidos para todas as amostras apresentaram-se no intervalo de 1 a $2 \%$, o que corresponde ao intervalo de valores observados para britas que varia de 0 a $4 \%$ [21]. Proporcionalmente, a resistência mecânica a compressão e massa específica aparente aumentaram com o acréscimo de vidro, conforme mostrado nas Figuras 4 e 5.

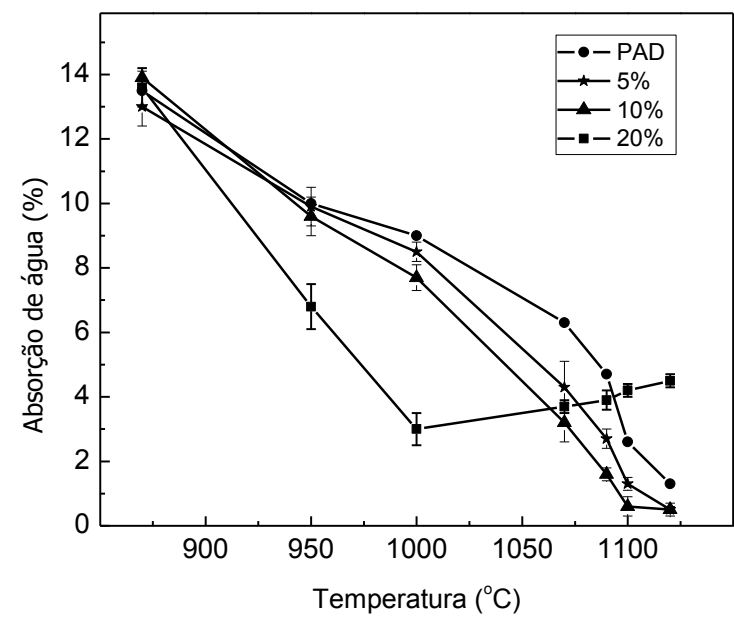

Figura 4: Absorção de água em função da temperatura de queima para massas contendo de 0 até $20 \%$ de vidro. 


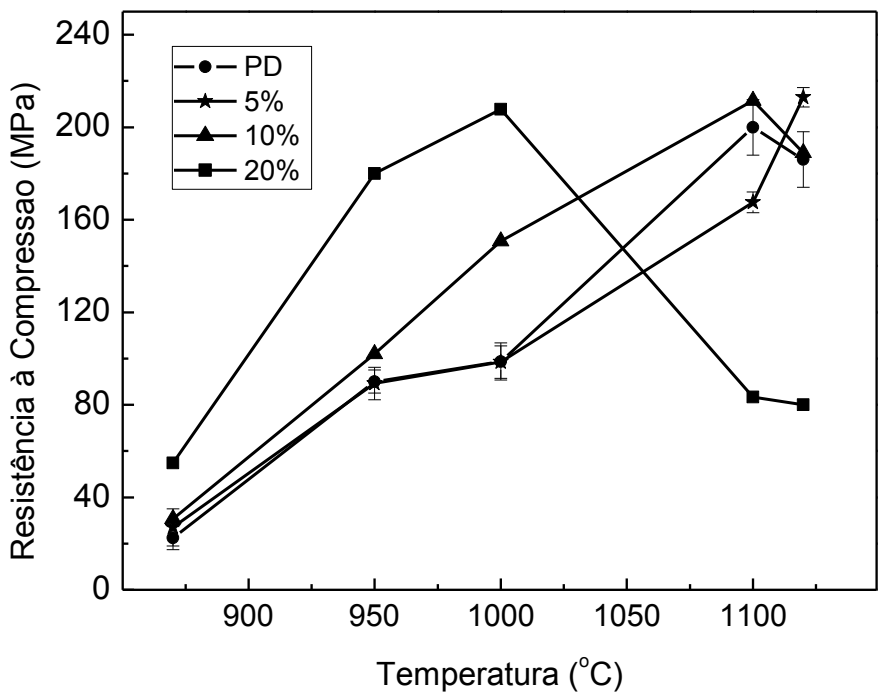

Figura 5: Resistência mecânica a compressão em função da temperatura de queima para massas contendo de 0 até $20 \%$ de vidro.

\section{CONCLUSÕES}

Os resultados tecnológicos das argilas estudadas comprovam que é possível produzir agregado sintético a partir das argilas estudadas.

Os resultados obtidos se aproximam de britas de uso corrente na construção civil que é 160 a $300 \mathrm{MPa}$. A análise dilatométrica foi fundamental para definir as temperaturas em que ocorrem o início e o fim da sinterização das massas, sobretudo a sua curva de densidade, conforme apresentado na Figura 4.

Foi observado nos testes de laboratório, que à medida que se acrescentou o vidro, a absorção de água reduziu e proporcionalmente, a resistência mecânica e massa específica aumentaram em todas as formulações, exceto a massa contendo $20 \%$ de vidro que a partir de $1000{ }^{\circ} \mathrm{C}$ experimentou o aumento de volume devido a maior formação de fase liquida que impediu a saída de gases provocando eventual inchamento.

$\mathrm{Na}$ análise dilatométrica a temperatura encontrada de máxima densificação para massas contendo $20 \%$ de resíduo foi próxima de $900{ }^{\circ} \mathrm{C}$, enquanto nos testes de laboratório os melhores resultados foram obtidos a $100{ }^{\circ} \mathrm{C}$. Esta diferença se deve a diferenças entre o tipo de forno, condições de queima e dimensões dos corpos de prova.

Na temperatura de $1000{ }^{\circ} \mathrm{C}$ a massa contendo $20 \%$ de vidro apresentou a mesma resistência mecânica (200 $\mathrm{MPa})$ e absorção de água (3\%) em relação as demais massas que obtiveram os mesmos resultados na temperatura próxima a $1100{ }^{\circ} \mathrm{C}$, conforme apresentado nas Figuras 7 e 8 , o que favorece a redução dos custos de processo de produção de agregados em função da redução da temperatura de queima.

A utilização do vidro poderá reduzir o impacto ambiental provocado pelo vidro que é descartado em lixos urbanos.

\section{AGRADECIMENTOS}

Os autores agradecem à CAPES, CNPQ e FAPITEC/SE pelo suporte financeiro, ao CMNano, DCEM e LPCM-NUPEG pelo uso dos laboratórios.

\section{BIBLIOGRAFIA}

[1] ROSSIGNOLO, J.A., Concreto Leve Estrutural, $1^{\mathrm{a}}$ Edição, Ed. Pini, São Paulo, 2011.

[2] CABRAL, E.M., SÁ, R.J. de, VIEIRA, R.K., VASCONCELOS, R. P., "Use of ceramic bodies in the production of synthetic aggregate calcined clay for use in concrete", Cerâmica, v.54, pp.404-410, 2008. 
[3] MORAVIA, W.G., OLIVEIRA, C. A. S., GUMIERI, A. G., et al., "Caracterização microestrutural da argila expandida para aplicação como agregado em concreto estrutural leve", Cerâmica, v.52, pp.193-199, 2006.

[4] FROTA, C.A., SILVA, A.C.L., "Estudo da viabilidade econômica para produção de agregado sinterizado de argila calcinada", Cerâmica,v. 59, pp. 509-517, 2013.

[5] SANTIS, B. C., ROSSIGNOLO, J.A., "Caracterização de massas cerâmicas do estado de São Paulo para produção de agregados leves para concreto", Cerâmica, v.59, pp. 350-359, 2013.

[6] SANTIS, B.C., ROSSIGNOLO, J.A., "Influence of calcined clay lightweight aggregates on the mechanical properties of structural concretes", Revista Matéria v. 20, n. 2, pp. 399-406, 2015.

[7] COSTAS, A.V., FRANCO-SAlinAS, C., O'SUllivanET, C., et al., "Up-Cycling Waste Glass to Minimal Water Adsorption/Absorption Lightweight Aggregate by Rapid Low Temperature Sintering: Optimization by Dual Process-Mixture Response Surface Methodology', Environ. Sci. Technol., v.48, pp. 7527-7535, 2014.

[8] JINSHAN, L., ZHANGYANG, L., CHANGHAO, P., et al., "Influence of particle size on sinterability, crystallization kinetics and flexural strength of wollastonite glass-ceramics from waste glass and fly ash", Materials Chemistry and Physics, v.148, pp.449-456, 2014.

[9] YU-LING, W., SHAO-HSIAN, C., GUAN-WEI, K., "Effect of waste glass addition on lightweight aggregates prepared from F-class coal fly ash", Construction and Building Materials, v.112, pp. 773-782, 2016.

[10] VOLLAND, S., KAZMINA, O., VERESHCHAGIN, V., et al., "Recycling of sand sludge as a resource for lightweight aggregates", Construction and Building Materials, v. 52, pp. 361-365, 2014.

[11] CHRISTOGEROU. A., KAVAS, T., "Synergy of boron containing solid astes and fructose for the production of light weight aggregates: microstructure and properties", Waste Biomass, v.5, pp. 1453-1461, 2016.

[12] KALIRAJAN, M., RANJEETH, R., VINOTHAN, R, et al., "Influence of glass waste in the microstructural evolution and crystallization kinetics of glass-ceramic glaze", Ceramics International, pp. 131-139, 2016.

[13] ABRELPE, Associação Brasileira de Empresas de Limpeza Pública e Resíduos Especiais. Disponível em http://www.abrelpe.org.br/Panorama/panorama2014.pdf, 2012.

[14] SHAYANA, X.A., "Value-added utilization of waste glass in concrete", Cement Concrete Res., v. 34, pp. 81-88, 2004.

[15] ANDREOLA, F., BARBIERI, L., BONDIOLI, F., et al., "Recycling screen glass into new traditional ceramic materials", Int. J. Appl. Ceram. Technol., v.7, pp. 909-917, 2010.

[16] ANDREOLA, F., BARBIERI, L., LANCELLOTTI, I., et al., "Recycling of industrial wastes in ceramic manufacturing: State of art and glass case studies", Ceramics International, v.4, pp. 13333-13338, 2016.

[17] DONDI, M., CAPPELLETTI, P., D'AMORE, R., et al., "Lightweight aggregates from waste materials: Reappraisal of expansion behavior and prediction schemes for bloating", Construction and Building Materials, v.127, pp. 394-409, 2016.

[18] MORELLI, A., BALDO, J.B., "Barbotinas Cerâmicas Contendo Rejeito de Vidro Soda Cal Para Maturação em Baixa Temperatura”, Cerâmica Industrial, v.8, pp. 36-41, 2003.

[19] TEIXEIRA, S.R., "Reuse of glass cullet as aggregate for red ceramic clays", Revista Ciências Exatas, v.2, n. 2, 12-16, 2007.

[20] BOLTAKOV, N.V., FASEEVA, G.R., KABIROV, R.R., et al., "Utilization of inorganic industrial wastes in producing construction ceramics", Review of Russian experience for the years 2000-2015, 2016.

[21] ISAIA, G.C., Concreto: ensino, pesquisa e realizações, IBRACON, São Paulo, 2005.

[22] AMOROS, J.L., SANCHES, G., JAVIER, M.M., "Manual para el control de la calidad de materias primas arcillosas", ITC Instituto de Tecnologia Cerámica, 1998.

[23] ASTM C-20, 2005, Standard Test Methods for Apparent Porosity, Water Absorption, Apparent Specific Gravity, and Bulk Density of Burned Refractory Brick and Shapes by Boiling Water, 2005.

[24] ASSOCIAÇÃO BRASILEIRA DE NORMAS TÉCNICAS (ABNT). NBR 10832: Fabricação de Tijolo Maciço de Solo-Cimento com a Utilização de Prensa Manual. Rio de Janeiro, 1989. 
[25] MAHMOUD, S., BENNOUR A., SRASRA, E., et al., "Characterization, firing behavior and ceramic application of clays from the Gabes region in South Tunisia”, Applied Clay Science, v.135, pp. 215-22, 2017.

[26] ASTM C-837, 2006. Standard Test Method for Methylene Blue Index of clay, 2006.

[27] ASTM D4318, Standard test method for Liquid Limit, Plastic limit, and plasticity index of soils, 2010.

[28] NBR 6459, Solo Determinação do Limite de Liquidez, 1984.

[29] ZAIED F.H.; ABIDI, R., SHIMI, N.S., SOMARIN A.K., "Potenciality of clay raw materials form gram area (Norther Tunisia) in the ceramic industry”, Applied Clay Science, v.112-113, pp.1-9, 2015.

[30] RAHAMAN, M. N., Ceramic Processing and Sintering, 2nd Edition, Missouri, Taylor \& Francis, 782, 2003.

[31] CELIK, H., "Technological characterization and industrial application of two Turkish clays for the ceramic industry", Appl. Clay Sci., v.50, pp. 245-254, 2010.

[32] AlCÂNTARA, A.C., BELTRÃO, M. S., OLIVEIRA, H. A., et al., "Characterization of ceramic tiles prepared from two clays from Sergipe-Brazil", Applied Clay Science, v.39, pp.160-165, 2008.

[33] BOUSSEN, S., SGHAQIER, D., CHAABANI, F., et al., "Characteristics and industrial application of the Lower Cretaceous clay deposits (Bouhedma Formation), Southeast Tunisia: Potential use for the manufacturing of ceramic tiles and bricks", Appl. Clay Sci., v.123, pp. 210-221, 2016.

[34] MONTEIRO, S.N., VIEIRA, C.M.F., "Influence of firing temperature on the ceramic properties of clays from Campos dos Goitacazes, Brazil”, Applied Clay Science, v.27, pp. 229-234, 2004.

[35] SOLTAN, A.M, KAHL, W.A., Abd RAOOF, F., et al., "Lightweight aggregates from mixtures of granite wastes with clay", Journal of Cleaner Production, 117, 139-149, 2016.

[36] DONDI, M., IGLESIAS, C., DOMINGUEZ, E., et al., "The Effect of kaolin properties on their behavior in ceramic processing as illustrated by a range of kaolins from the Santa Cruz and Chubut provinces, Patagonia (Argentina)", Appl. Clay Sci., 40, 143-158, 2008.

[37] DONDI, M., FABBRI, B., GUARINI, G., "Grain-Size distribution of Italian raw materials for building clay products: a reappraisal of the Winkler diagram”, Clay Mineral, v.9, n.33, pp. 435-442, 1998.

\section{ORCID}

Herbet Alves de Oliveira

https://orcid.org/0000-0003-4159-6325

Zelia Soares Macedo

https://orcid.org/0000-0002-8577-7622

Cochiran Pereira dos Santos

https://orcid.org/0000-0001-9819-6002

Rosane Maria Pessoa Betânio Oliveira https://orcid.org/0000-0002-2726-1863

Edilson de Jesus

https://orcid.org/0000-0003-4239-6050 